\title{
LEAN MANUFACTURING EN PYMES
}

\section{LEAN MANUFACTURING IN SMEs}

Recepción: 02/05/2017

Aceptación: 17/09/2017

Publicación: 22/12/2017

Jorge Sanz Horcas ${ }^{1}$

Víctor Gisbert Soler ${ }^{2}$

1. Grado en Ingeniería Química (Universidad de Valencia). Máster Universitario en Ingeniería de la Organización y Logística (Universidad Politécnica de Valencia). (España). E-mail: jorsanho@epsa.upv.es

2. Doctor Ingeniero Industrial. Departamento de Estadística e Investigación Operativa Aplicadas y Calidad. Universidad Politécnica de Valencia. (España). E-mail: vgisber@eio.upv.es

Citación sugerida:

Sanz Horcas, J. y Gisbert Soler, V. (2017). Lean manufacturing en pymes. 3C Empresa: investigación y pensamiento crítico, Edición Especial, 101-107. DOI: <http://dx.doi.org/10.17993/3cemp.2017.especial.101-107/>. 


\section{RESUMEN}

El Lean Manufacturing es una metodología basada en las personas que se focaliza en mejorar los procesos eliminando todo aquello que no genera valor.

En el presente artículo se define la filosofía Lean Manufacturing nombrando algunas de sus técnicas básicas, objetivos y principios, con la intención de dar a conocer esta filosofía a las empresas que no han llevado a cabo su implantación, en concreto a las Pymes.

\section{RESUMEN}

Lean Manufacturing is a people-based methodology that focuses on improving processes by eliminating all that does not generate value.

In this article the Lean Manufacturing philosophy is defined naming some of its basic techniques, objectives and principles, with the intention of making known this philosophy to the companies that have not carried out their implantation, specifically to SMEs.

\section{PALABRAS CLAVE}

Lean Manufacturing, Pymes, desperdicios, valor añadido, competitividad.

\section{KEY WORDS}

Lean Manufacturing, Smes, _, value added, competitiveness. 


\section{INTRODUCCIÓN}

En la actualidad las empresas se enfrentan a un mercado cada vez más competitivo y buscan técnicas organizativas para hacer frente a éste. Una de estas técnicas es el Lean Manufacturing, con un gran potencial y recomendada por muchas de las empresas que han llevado a cabo su implantación.

El Lean Manufacturing tiene su origen en la empresa automovilística Toyota (Japón) tras el desarrollo del sistema productivo Just In Time (JIT) en los años 50 y, con el paso de los años, se ha configurado un modelo de técnicas de mejora de los procesos llevando a empresas a lo más alto de la competencia industrial.

Se puede decir que el modelo Lean consiste en la aplicación sistemática y habitual de un conjunto de técnicas de fabricación que buscan la mejora de los procesos productivos a través de la reducción de todo tipo de "desperdicios", definidos éstos como los procesos o actividades que usan más recursos de los necesarios. Para llevar a cabo este modelo con éxito es necesario un cambio de cultura en la organización y la implicación de los directivos, mandos intermedios y operarios. Los beneficios obtenidos en una implantación Lean son evidentes y están demostrados.

En la actualidad, el interés por el Lean Manufacturing está en continuo crecimiento pero prácticamente en la totalidad de los casos por las grandes empresas, siendo éste más desconocido entre las pequeñas y medianas empresas. Esto es debido a que en algunas empresas se considera difícil su implantación, ya que para que sea efectiva es necesario la implicación de todo el personal de la empresa, desde los directivos hasta los operarios y, en muchas ocasiones el problema principal está en la resistencia al cambio de los trabajadores y la ausencia de liderazgo y convicción de los beneficios por parte de la dirección de la empresa. La implicación de la alta dirección y sus acciones de motivación y comunicación con todos los niveles de la empresa resultan fundamentales para alcanzar unos resultados positivos en la implantación.

\section{2. ¿QUÉ ES LEAN MANUFACTURING?}

Lean Manufacturing es un modelo de gestión basado en las personas, que define la forma de mejora y optimización de producir bienes y servicios, focalizándose en identificar y eliminar todo tipo de "desperdicios", definidos éstos como toda actividad, que supone el consumo de algún recurso (equipos, materiales, espacio, tiempo, personas,...), que no aporta valor alguno al producto o servicio que está generando.

Dentro de la empresa, estas actividades se identifican como los " $7+2$ desperdicios" que son los siguientes:

- Sobreproducción

- Sobreproceso

- Inventario 
- Transporte

- Movimientos

- Esperas

- Defectos

- Habilidades

- Exceso reuniones y mails

Lean tiende a eliminar todas las actividades que no agregan valor al cliente y, para llevarlo a cabo aplica una serie de técnicas sistemáticas y habituales que cubren todas las áreas operativas de fabricación: flujo interno de producción, puestos de trabajo, mantenimiento, calidad, aprovisionamiento, etc.

La filosofía Lean es una filosofía dinámica, es decir, que continuamente está buscando formas de realizar las cosas de la manera más fácil, flexible y al mínimo coste.

Su objetivo final es el de generar una nueva cultura en la organización basada en la comunicación, la mejora y el trabajo en equipo, apoyada con la aplicación de técnicas surgidas del estudio a pie de máquina.

\section{OBJETIVOS DE LAS EMPRESAS LEAN MANUFACTURING}

Algunos de los objetivos que persiguen las empresas que llevan a cabo la implantación de Lean pueden ser los siguientes:

- Diseñar para "fabricar".

- Reducir los tiempos de preparación de máquinas para incrementar la flexibilidad y disminuir los plazos de ejecución.

- Lograr una distribución de la planta que asegure un bajo inventario, minimice recorridos y facilite el control directo por visibilidad.

- Usar la tecnología para disminuir la variabilidad del proceso.

- Conseguir que sea fácil fabricar el producto sin errores.

- Organizar el lugar de trabajo para eliminar tiempos de búsquedas.

- Formar a los trabajadores para facilitar la motivación, polivalencia y multidisciplinariedad.

- Garantizar que el personal de línea sea el primero en intentar solucionar los problemas.

- Conservar y mejorar el equipo existente antes de pensar en nuevos equipos. Usar intensivamente el mantenimiento preventivo implicando a todos los empleados.

- Incrementar la frecuencia de entregas de los productos. 
- Conseguir que la detección de fallos se realice en la fuente creando mecanismos sencillos que detecten inmediatamente los problemas.

- Garantizar que todas las personas estén regularmente informadas sobre las necesidades de los clientes, su grado de satisfacción y de los métodos a utilizar para su satisfacción.

\section{PRINCIPIOS DEL LEAN MANUFACTURING}

Los principios más frecuentes asociados al sistema, desde el punto de vista del "factor humano" y de la manera de trabajar y pensar, son:

- Trabajar en la planta y comprobar las cosas in situ.

- Formar líderes de equipos que asuman el sistema y lo enseñen a otros.

- Interiorizar la cultura de "parar la línea".

- Crear una organización que aprenda mediante la reflexión constante y la mejora continua.

- Desarrollar personas involucradas que sigan la filosofía de la empresa.

- Respetar a la red de suministradores y colaboradores ayudándoles y proponiéndoles retos.

- Identificar y eliminar funciones y procesos que no son necesarios.

- Promover equipos y personas multidisciplinares.

- Descentralizar la toma de decisiones.

- Integrar funciones y sistemas de información.

- Obtener el compromiso total de la dirección con el modelo Lean.

A estos principios hay que añadir los relacionados con las medidas operacionales y técnicas a usar:

- Crear un flujo de proceso continuo que visualice los problemas a la superficie.

- Utilizar sistemas "Pull" para evitar la sobreproducción.

- Nivelar la carga de trabajo para equilibrar las líneas de producción.

- Estandarizar las tareas para poder implementar la mejora continua.

- Utilizar el control visual para la detección de problemas.

- Eliminar inventarios a través de las diferentes técnicas JIT.

- Reducir los ciclos de fabricación y diseño.

- Conseguir la eliminación de defectos.

\section{TÉCNICAS BÁSICAS DEL LEAN MANUFACTURING}

El Lean Manufacturing posee una gran variedad de técnicas y con distintas finalidades, que se pueden implantar de forma independiente o conjunta, atendiendo a las necesidades específicas de cada caso. Para llevar a cabo la aplicación de éstas es necesario un diagnóstico previo del problema. 
Algunas de las técnicas Lean son: 5S, SMED, Estandarización, TPM, Control Visual, Jidoka, Heijunka, Kamban, etc.

La gran variedad de técnicas y la dificultad de algunas de ellas en su implantación hace que ciertas empresas no den el paso de llevar a cabo una implantación Lean, además del gran coste económico que suponen algunas, que únicamente las grandes multinacionales son capaces de implantar.

Pero hay una serie de técnicas Lean que por su enfoque práctico y el sentido común, se podría decir que deberían ser de obligado cumplimiento en cualquier tipo de organización, desde las grandes multinacionales hasta las PYMEs, y son las siguientes:

- Las 5S. Técnica utilizada para la mejora de las condiciones del trabajo de la empresa a través de una excelente organización, orden y limpieza en el puesto de trabajo.

- SMED. Sistemas empleados para la disminución de los tiempos de preparación.

- Estandarización. Técnica que persigue la elaboración de instrucciones escritas o gráficas que muestren el mejor método para hacer las cosas.

- TPM. Conjunto de múltiples acciones de mantenimiento productivo total que persigue eliminar las perdidas por tiempos de parada de las máquinas.

- Control visual. Conjunto de técnicas de control y comunicación visual que tienen por objetivo facilitar a todos los empleados el conocimiento del estado del sistema y del avance de las acciones de mejora.

Estas técnicas son indispensables en cualquier empresa que pretenda competir en el mercado actual y con ellas se obtienen unos resultados muy positivos. Por estas razones, y por la facilidad de implantación en relación con otras técnicas Lean, es aconsejable que las PYMEs lleven a cabo su implantación, de forma independiente o conjunta.

\section{CONCLUSIONES}

En esta publicación se ha pretendido mostrar la filosofía Lean desde las técnicas básicas para que las pequeñas y medianas empresas que no sabían de ésta las conozcan y se interesen para llevar a cabo su implantación.

Lean Manufacturing es una filosofía que se basa en las personas y que nace de la observación directa de los problemas con la finalidad de hacer bien las cosas y eliminar todo aquello que no aporta valor. La efectividad de las herramientas utilizadas en Lean están demostradas y hacen que las empresas sean más competitivas.

Para asegurar el éxito en la implantación es imprescindible la implicación y la buena actitud de todo el personal de la organización, desde la dirección hasta los empleados. 


\section{REFERENCIAS BIBLIOGRÁFICAS}

Hernández Matías, Juan Carlos; Vizán Idoipe, Antonio. Lean Manufacturing. Concepto, técnicas e implantación [Libro electrónico]. Madrid: EOI, Escuela de Organización Industrial Universidad Politécnica de Madrid, 2013. [Consulta: 10 mayo 2017]. Disponible en: https://www.eoi.es/es/savia/publicaciones/20730/lean-manufacturingconcepto-tecnicas-e-implantacion 